\title{
Lenguas en desplazamiento. Representaciones literarias de problemáticas lingüísticas en la e(in)migración italiana en Argentina
}

\author{
Fernanda Bravo Herrera \\ CONICET - Instituto de Literatura \\ Argentina «Ricardo Rojas»
}

\section{Resumen}

El objetivo de este trabajo es analizar en una selección significativa de textos italianos y argentinos diferentes representaciones de las problemáticas lingüísticas vinculadas con el fenómeno de la e(in)migración italiana en Argentina. La lectura, desde la literatura comparada y desde la sociocríti$\mathrm{ca}$, se propone reconstruir las varias perspectivas ideológicas que configuran los encuentros culturales provocados por desplazamientos migratorios, que determinan las reestructuraciones identitarias y son visibles en los reacomodamientos lingüísticos, a través de los conflictos o de sus resoluciones. De esta manera se espera delinear, en la inscripción de las representaciones y estilizaciones lingüísticas, una lectura semiótica de las reconfiguraciones identitarias en vinculación con los procesos migratorios.

\section{Palabras clave}

· Migración · identidad · alteridad · representaciones · ideologías 


\section{Abstract}

The objective of this paper is to analyze in a significant selection of Italian and Argentinean texts the different representations of linguistic problems linked to the phenomenon of Italian e(in)migration in Argentina. Reading, from comparative and socio-critical literature, aims to reconstruct the various ideological perspectives that make up cultural encounters caused by migratory movements, which determine identity restructuring and are visible in linguistic rearrangements, through conflicts or their resolutions. In this way, it is expected to outline, in the inscription of the linguistic representations and stylizations, a semiotic reading of the identity reconfigurations in connection with the migratory processes.

\section{Key words}

- Migration · identity · otherness - representations · ideologies

\section{Premisas}

Este trabajo se propone analizar algunas representaciones significativas de las problemáticas lingüísticas vinculadas con los desplazamientos migratorios de Italia a Argentina en un corpus de textos pertenecientes a los dos espacios geoculturales, a fin de identificar y reconstruir las posiciones ideológicas legibles en dichas representaciones y estilizaciones ${ }^{1}$. Para el abordaje, sobre todo del corpus y de las problemáticas analizadas, se opera con el concepto de e(in)migración, para resaltar el carácter complejo, poliédrico, polífono, plurilingüe e intergeneracional del fenómeno y para realizar una lectura comparada de genotextos, es decir, núcleos constantes de productividad semántica y significativa que pueden declinarse con variaciones y divergencias según las perspectivas ideológicas. La conformación identitaria bajo el signo de la e(in)migración constituye uno de los núcleos problemáticos centrales del fenómeno, sea en su movimiento afirmativo de identificación y pertenencia, como en la señalización de las diferencias y distancias desde la alteridad, la extrañeidad y la extranjeridad. Este genotexto puede inscribirse, en las diferentes textualidades, modelizado por múltiples ideologías y declinado en relación con otras problemáticas y cuestiones. Entre estas, la relativa a los contactos entre lenguas es fundamental y clave en el abordaje del fenómeno e(in)migratorio no solamente desde la especificidad de los estudios lingüísticos. Es necesario recordar que la lengua organiza, manifiesta y manipula la comprensión del mundo, 
las formas de habitarlo y nombrarlo, la organización del pensamiento, las relaciones con otros y las proyecciones de la subjetividad, y que el lenguaje es, por ello, visibilización de estructuras imaginarias. En los desplazamientos demográficos y en los contactos culturales, las interacciones lingüísticas condensan estas problemáticas, evidenciando, en los conflictos y sus posibles resoluciones, cómo lenguaje y vida están indisolublemente vinculados y modelados desde lo social. A partir de estas premisas, la lectura comparada busca identificar en las diferentes representaciones de los contactos lingüísticos en situaciones determinadas por la $e$ (in)migración italiana en Argentina las perspectivas ideológicas que modelizan y (re) significan social y culturalmente a dicho fenómeno y a los sujetos que participan en las dos orillas. El recorrido atiende, en el primer apartado, las representaciones de la complejidad lingüística a partir de la estratificación dialectal, la debilidad del italiano como lengua nacional y la fuerte caracterización de los e(in)migrantes desde su analfabetismo e impreparación; $y$, en el segundo, las representaciones de algunos mecanismos y resoluciones de los contactos y conflictos lingüísticos, tendientes a la conservación y a los desplazamientos que determinan y manifiestan, a su vez, las reconfiguraciones identitarias.

\section{Los mosaicos lingüísticos y las torres de Babel}

El mapa lingüístico italiano, especialmente durante los flujos migratorios del siglo XIX, conformaba un mosaico complejo de dialectos y variedades lingüísticas regionales que evidenciaba, por una parte, un horizonte identitario múltiple, fragmentario y estratificado y, por otra parte, era índice de una conformación de pertenencia e identificación constituida desde realidades locales diferenciadas sin que, en algunos casos, se alcanzase una estructuración nacional unificadora. Esto significa que la reconfiguración identitaria de los sujetos, tendiente a alcanzar la integración y la definición después del desplazamiento migratorio, no tenía que realizarse solamente en la relación dialéctica con el nuevo espacio adoptado, es decir Argentina, sino también con el espacio ausente, fragmentado, estratificado, y con otros italianos en Argentina, que señalaban múltiples alteridades, (dis)continuidades y diferencias. La pertenencia y la identificación con una comunidad local podían ser, en algunos sujetos, las únicas formas de estructuración identitaria, sin que en la misma opere, antes de la emigración, una instancia de apelación nacional. La frase de Massimo D'Azeglio, «Fatta l'Italia, bisogna fare gli italiani», además de describir la fragmentación socio-cultural italiana en el momento de alcanzar la independencia y la unificación 
del Estado, resume un proyecto político de construcción identitaria que, en muchos casos, se realizó fuera del territorio, asumiendo la condición de migrantes, de italiani all'estero, en el reconocimiento de coincidencias por sobre las diferencias, impulsados por políticas estatales tendientes a reforzar - o crear - lazos simbólicos de pertenencia. Esto se debió a que

(...) dalla fine dell'Ottocento, le autorità italiane, laiche ed ecclesiastiche, fanno di tutto per evitare che gli emigranti perdano la loro italianità. Lo Stato italiano e la Chiesa cattolica cercano di organizzare strutture in grado di preservare la cultura e la fede dei propri "figli» all'estero. Soprattutto nelle Americhe i funzionari diplomatici e i missionari si prodigano per salvaguardare l'italianità degli emigrati e finiscono per crearla, fondando e sovvenzionando scuole e associazioni italiane e promuovendo l'attività di organizzazioni come la Dante Alighieri. (Sanfilippo, 2005: 207-208)

Esta realidad ha sido representada en numerosos textos, en los cuales, además de describir y señalar las proveniencias geo-socioculturales de los e(in)migrantes a partir de su lengua, se pone en evidencia la multiplicidad lingüística del país de proveniencia, su fragmentación cultural, las divisiones que obstaculizan el proyecto de unificación nacional. La presencia del dialecto, entonces, no se concibe en su potencialidad comunicativa sino en sus limitaciones, como indicio de una alfabetización incompleta o ausente y como factor determinante de un fracaso político de organización del Estado-nación según principios asentados en la Modernidad. El dialecto se vincula con la cultura oral, por lo que en las representaciones se muestra a sujetos carentes de la capacidad de escritura-lectura y, por ello, muchas veces, víctimas de estafas. Si bien Gesualdo Bufalino en «Messaggi di "lingue tagliate"», incluido en La luce e il lutto (1988), se refiera a la emigración hacia Alemania, es significativa también para América la descripción de la voluntad de comunicación por escrito de los emigrantes, no obstante el analfabetismo de quienes, «riluttanti a dividere con uno scrivano estraneo e venale i segreti della propia intimità (...), ricorsero, per corrispondere, a un linguaggio di convenzione, un sistema di pittografie in sequenza, il cui senso risultasse intelligibile al destinatario» (Bufalino, 2006: 1274). La comunicación epistolar resulta, pues, espacio de visibilización de las realidades lingüísticas de los e(in)migrantes, tanto de su adscripción dialectal, de las interferencias, cristalizaciones y desplazamientos lingüísticos, como de la oralidad y del analfabetismo. Esto es relatado por Syria Poletti en Gente conmigo (1962), cuya protagonista, alter ego de la autora, asume el «extraño oficio» de escribir cartas y contratos por cuenta de quienes no podían hacerlo. La problemática planteada por Poletti supera las derivadas por la incomunicabilidad lingüística, pues narra la fragmentación social y familiar provocada por los desplazamientos migratorios:

Abuela y yo éramos tan pobres que para comer teníamos que cobrar unos centavos por cada carta que escribíamos. Y esperábamos clientes como los esperan los abogados y los funebreros. (...) A las madres que tenían hijos en América tampoco les cobrábamos nada. Era nuestra norma. En esas cartas iba más sangre que tinta, más silencio que palabras. Imposible cobrar todo eso. En las cartas para América no había que buscar giros difíciles ni retórica de actualidad, porque todas decían o callaban las mismas cosas. (Poletti, 1972: 10) 
En las representaciones de los emigrantes italianos caracterizados a partir de los dialectos - especialmente cuando el dialecto es la única lengua que manejan y esto constituye el principal rasgo distintivo e identificatorio - se denuncia un doble fracaso: el del proyecto de unidad italiana del Risorgimento y el del fare l'America. La limitación dialectal acompaña, en consecuencia, la fragmentación política italiana y la incapacidad de emprender, tanto desde la perspectiva italiana como de la argentina, una gesta $e($ in)migratoria heroica, una integración social y un rescate moral en América. Giovanni Graziani en La emigrazione italiana nella Repubblica Argentina (1905) denuncia el mal de la «ignorancia» que provoca que los emigrantes renuncien, por vergüenza, a la propia cultura e, incapaces de comunicarse con otros compatriotas con una lengua común de pertenencia nacional por el uso exclusivo del dialecto, abandonen la lengua materna, sin aprender el italiano, percibiendo la extranjeridad y la alteridad dentro de la misma comunidad italiana. De esta manera se acentúa su fragmentación y disolución, pues los $e($ in)migrantes se encuentran en una situación de debilidad y precariedad social en Argentina, por lo que, al no poder mejorar sus condiciones socio-económicas, se determina, en consecuencia, el fracaso del proyecto de emigración:

Gli emigranti nostri diretti in America non conoscono l'italiano: ciascuno sa il dialetto della sua regione. Un Siciliano ed un Veneto sono fra loro stranieri. Non potrà mai iniziarsi fra loro quello scambio di parole che riuscirebbe talora a diradare le tenebre di parte della ignoranza reciproca; essi rimarranno muti perchè, già, parlando, non si comprenderebbero: non può stabilirsi fra i due quella corrente di idee che talora, cozzando fra loro nel fervore della discussione, potrebbero portare in campo una verità non saputa, non si può stringere fra loro quel legame di simpatia e di solidarietà che è il gran conforto nel dubbio, nello scoramento, nella sventura. Non potranno apprezzare le virtù e le glorie delle loro terre: ciascuno non conoscerà che le miserie della propia e null'altro: l'Italia rimarrà così sempre piccola nelle loro menti, chiusa sempre dentro di quell'orizzonte ristretto, che non si allargherà più. - Giunti in America rimangono storditi davanti al fasto delle grandi capitali. - Le considerazioni si ripetono. Saranno i deboli, i poveri di spirito. Occuperanno il gradino più umile della scala sociale. (Graziani, 1905: 85)

El desconocimiento de la lengua nacional revela para Edmondo De Amicis la carencia de la alfabetización y, por tanto, la imposibilidad de conocer los riesgos de emigrar. Sobre esta última falencia, la lectura que realiza De Amicis de la emigración italiana coincide con la de Luigi Barzini, quien denuncia la falta de preparación y formación de los emigrantes, porque ignoran no solamente todo lo relativo al país de destinación, sino también del propio. Giovanni Graziani apunta, por su parte, como causas del fracaso de los emigrantes italianos en Argentina a «la mancanza di preparazione e di organizzazione nonchè l'assoluto abbandono in cui li lascia la madre-patria (...) insieme con la profonda ignoranza che caratterizza» (Graziani, 1905: 86). Antonio Gramsci, en Quaderni dal carcere, denunció que la falta de información e interés en conocer la realidad vinculada con la emigración no se limitaba a quienes emigraban sino que incluía también a los literatos e intelectuales (Gramsci, 1977: 2254). De esta manera la emigración, en vez de transformarse en un hecho de sublimación y rescate social, o en una pacífica conquista por medio de la colonización y la expansión, conllevaba el abandono de las propias características y diferencias culturales, entre ellas la lingüística, para adaptarse al nuevo espacio.

En Sull'Oceano (1889) de Edmondo De Amicis las descripciones de los emigrantes 
que se dirigen a Argentina y a Uruguay ofrecen un cuadro de las diferentes realidades de una Italia miserable, triste espectáculo no solo político-social sino incluso a nivel humano por las condiciones de pobreza y desamparo de estos protagonistas anónimos ${ }^{2}$. Las caracterizaciones de los emigrantes según su lugar de proveniencia y sus formas dialectales son constantes en el texto y funcionan como categorías que permiten delinear un completo inventario de la hemorragia humana, en última instancia, un análisis del estado del país que sufría esta fuga en la totalidad de su territorio, dividido pese a la Unidad política. Así, en el capítulo «L'Italia a bordo» la descripción de los emigrantes, especificando su origen, funciona elípticamente como el diseño del mapa geográfico y demográfico de Italia, como condensación simbólica y significativa del Estado en su fragmentación regional ${ }^{3}$. Esta fuerte conformación identitaria de los emigrantes desde su pertenencia regional se manifiesta también lingüísticamente mostrando - como se evidencia en el capítulo «Sul tropico del Cancro» (De Amicis, 1996: 76-77) — la paradoja de la incomunicabilidad entre los miembros de un Estado unificado y la necesidad imperiosa de una lengua nacional. Esto no solamente respondía a un proyecto político de organización del Estado nacional y de definición de parámetros de identidad y pertenencia, sino también a una reafirmación y defensa de un horizonte de pertenencia más allá del territorio y de las fronteras estatales. Este proyecto reconocía la debilidad de los dialectos de los emigrantes frente a la imposición de una lengua nacional, sea «materna» o de adopción. Se trataba, entonces, de una manera de preservar la identidad, aun en condiciones de desplazamientos migratorios, a partir de la valorización de una única lengua que evitara la conformación de interlenguas o interferencias:

Ma bisognava sentire che vocabolario: era il primo saggio ch'io intendevo della strana lingua parlata dalla nostra gente del popolo dopo molti anni di soggiorno nell'Argentina, dove, col mescolarsi ai figli del paese, e a concittadini di varie parti d'Italia, quasi tutti perdono una parte del proprio dialetto e acquistano un po' d'italiano, per confonder poi italiano e dialetto con la lingua locale, mettendo desinenze vernacole a radicali spagnuole, e viceversa, traducendo letteralmente frasi proprie dei due linguaggi, le quali nella traduzione mutan significato o non serban più alcuno, e saltando quattro volte, nel corso di un periodo, da una lingua all'altra, come deliranti. Trasecolando gli udii dire si precisa molta plata per "ci vuol molto danaro" guastar capitali per «spender capitali», son salito con un carigo di trigo per «son partito con un carico di grano». E in quest'orribile gergo tirava via a dar addosso alla Camera dei Deputati, al governo atrasado (rimasto addietro), al popolo di mendigos, e perfino ai monumenti d'arte, dicendo che, nel ripassare per Milano, aveva trovato il Duomo molto più piccolo di come l'aveva nella mente. (De Amicis, 1996: 37)

La lengua, es necesario recordar, constituía un problema clave para De Amicis, quien en Lídioma gentile (1905), además de insistir sobre la necesidad de su estudio con una preocupación inscripta fuertemente en el ejercicio de la didáctica, vinculó estrechamente a la lengua con la conformación identitaria colectiva e individual:

L'amiamo perchè è la nostra nutrice intellettuale, il respiro della mente e dell'animo nostro, l'espressione di quanto è più intimamente proprio della nostra indole nazionale, l'immagine più viva e più fedele e quasi la natura medesima della nostra razza. L'amiamo perchè è il vincolo più saldo della nostra unità di popolo, l'eco del nostro passato, la voce del nostro avvenire, verbo non solo, ma essenza dell'anima della patria. (De Amicis, 1905: 3-4) 
Luigi Barzini, en su artículo «I figli degli italiani», publicado en Corriere della Sera y recogido sucesivamente en L'Argentina vista come è (1902), comparte la posición de De Amicis de identificación de la lengua como caracterizadora de la identidad de un pueblo, de tal modo que la misma actúa como elemento de cohesión y como vínculo con la patria aun en condiciones de exilio o emigración. De esta manera, el mantenimiento de la lengua implica la supervivencia de una patria más allá de las fronteras territoriales, constituida por elementos culturales y de carácter supranacional. Así, la lengua se concibe como «segno primo di riconoscimento» (Barzini, 1902: 175) y "parlare la propia lingua all'estero significa respirare un po d'aria della Patria (...); poche parole del nostro idioma bastano a far compiere all'anima un rápido viaggio in luoghi amati e lontani, e farla tornare più lieta e serena» (Barzini, 1902: 175).

Esta vinculación entre lengua y nación fue planteada también, desde el nacionalismo, por Aníbal Latino (José Ceppi) en El concepto de la nacionalidad y de la patria, diferenciándose sin embargo de Edmondo De Amicis, pues sostuvo que «está comprobado que los dialectos no son un obstáculo para la existencia de las naciones» (Latino, 1914: 239) y afirmó la coexistencia de dialectos y lengua nacional, subordinando, sin embargo, los dialectos a la lengua, especialmente en la práctica literaria, pues

allí donde hay varios dialectos, como en Italia y España, ó varios idiomas considerados como tales por su abolengo ó por el hecho de hablarlos también otros pueblos de otras naciones, hay una lengua oficial que estudian y hablan, y por consiguiente entienden, la mayor parte de los habitantes. (Latino, 1914: 239-240).

Luigi Barzini, por su parte, en su defensa de la italianidad señaló, como De Amicis, la necesidad del italiano como lengua única y como «il vincolo più forte con la Patria d'origine» (Barzini, 1902: 177). Desde esta perspectiva, el predominio de los dialectos y la ausencia del italiano conducen a la desintegración de la comunidad de emigrantes, sea por la presión de la lengua del país y por las políticas de nacionalización, sea por la incomunicabilidad entre aquellos que provienen de diferentes regiones lingüísticas. Barzini, además, señaló que el uso exclusivo de los dialectos caracterizaba a la mayoría de los emigrantes, que no eran cultos, es decir, alfabetizados, lo que determinó una doble manifestación de extranjeridad, una en relación con el país de recepción del flujo migratorio, y otra en su vinculación con otros emigrantes aun proviniendo del mismo país. Los dialectos, entonces, construían fronteras entre los mismos emigrantes cuando no había un acompañamiento de la lengua nacional de origen, por lo que para alcanzar la integración los dialectos se desplazaban a favor de la lengua del país que los recibía, abandonando así una parte fundamental de la estructura identitaria de origen. Así, Barzini lo señaló:

Ebbene, quanti italiani in Italia parlano italiano?

I colti tutti; ma noi sappiamo che disgraziatamente essi non formano la maggioranza nella massa emigratrice. Gli altri parlano ligure, parlano siciliano, romagnolo, lombardo, napoletano, piemontese, ma non italiano. La nostra lingua non va più al nord di Pistoja e più al sud di Roma. Un povero lavoratore abruzzese si troverà di fronte ad un suo compagno d'emigrazione ligure, come di fronte ad uno straniero. Mancherà lo slancio dell'affratellamento. Essi non potranno parlarsi, perciò non potranno conoscersi. E per amarsi bisogna conoscersi. Ecco forse la prima e più grave ragione della dispersione, della scissione e della discordia. 
(...) L'emigrato che non conosce che il suo dialetto, dovrebbe studiare l'italiano come una lingua nuova; è assurdo. Quando debe imparare una nuova lingua impara la più utile: quella del paese. (Barzini, 1902: 176)

La presencia de numerosas lenguas no solamente se registró en Italia durante el fenómeno de los grandes flujos migratorios, sino también en Argentina, sobre todo en la ciudad de Buenos Aires, que era caracterizada en numerosos textos por su cosmopolitismo y su condición babélica. Estas características no siempre tenían una valencia positiva vinculada con el progreso y el crecimiento demográfico, sino más bien una evaluación negativa que señalaba el caos y el peligro de disgregación social. La mirada se imponía desde el nacionalismo que buscaba conservar un patrimonio castizo que se consideraba verdadero baluarte de civilización, amenazada por la salvaje y bárbara inmigración. Esta es la descripción que ofrece Héctor Pedro Blomberg de Buenos Aires en Las puertas de Babel (1920), tal como señala Manuel Gálvez en la introducción:

Los puertos de Buenos Aires, y los barrios que los rodean: la Boca, el Dock Sur, el Paseo de Julio, son las puertas de Babel. Por ellos se entra en la ciudad monstruosa e inquietante donde todos los idiomas del mundo y todas las razas se confunden y mezclan. Arriba está la ciudad rica y poderosa. Abajo, es decir en las puertas de Babel, se aglomera la caravana de los parias, la turba sucia y doliente que arrastra por los puertos y los mares su desolación y su miseria. (Gálvez, 1920: 9)

La Boca, protagonista de la novela de Blomberg y mencionada por Manuel Gálvez, se configuraba para el italiano José Ceppi (Aníbal Latino) en Cuadros sud-americanos (1888) como el espacio más representativo de la inmigración italiana en la ciudad de Buenos Aires en una doble dimensión que comprendía, por una parte, la movilidad por su apertura como puerto y, por otra, el asentamiento y la integración como barrio eminentemente conformado por inmigrantes. La perspectiva positiva apuntaba al crisol de razas y no a la conformación de una Babel caótica y desintegradora:

en tierra los acentos de varias lenguas y dialectos de Italia que cruzan en todas las direcciones, (...) formando un espectáculo alegre, en el que la vida de tierra y la vida de mar se confunden, como en una gran fiesta de alianza, de fraternidad y de paz. (Ceppi, 1888: 24)

La visión babélica de la ciudad se inscribe en Libro extraño. Don Manuel de Paloche (1899) de Francisco A. Sicardi, y aunque en este texto se registra el caos urbano, esta caracterización no deja de resaltarse positivamente por el crecimiento urbano y moderno que determinaba el abandono de la vida rural. La torre de Babel estaba causada, entonces, por una modernización que, no obstante todos los defectos, significaba evolución, progreso y trabajo. Sicardi, al describir la ciudad de Buenos Aires destacó que en ella «se habla un extraño lenguaje, una mezcla de todos los idiomas. Al fin se mueve la enorme caravana en medio de un pueblo vigoroso, que parece llevar en su sangre los gérmenes sanos de todas las razas» (Sicardi, 1899: 8). Desde el humor y la ironía de Sicardi el encuentro de D. Manuel Paloche con el guardián italiano, con el cual no logra comunicarse por las diferencias lingüísticas, no implica un conflicto o una situación violenta para el argentino, como lo había sido para el gaucho Martín Fierro el no-diálogo con el napolitano, sino 
una manifestación del crecimiento del país, de la confluencia de razas, de la evolución de «esta ciudad políglota y enorme, sacudida por el vértigo de la creación y del crecimiento" (Sicardi, 1899: 19). El cosmopolitismo, al final, se revela en su faceta negativa como manifestación de un proceso dialéctico en curso, sin que se llegue a una síntesis unificadora que equilibre las diferencias. La mezcla y los contrastes no se resuelven todavía, en forma positiva, con la creación de una nueva raza civilizadora, que es la utopía que se auspicia Manuel Paloche, cual Quijote argentino, pues el país «es un hervidero de razas. De este crisol (...) ha de salir la verdad civilizadora. Todavía no estamos en ella» (Sicardi, 1899: 28), por lo que

al final le parecía que quedaba, á pesar de todo, mucho que hacer. Por lo pronto, él no comprendía cómo ha podido llegar á creer en la grandeza de un país cuyo idioma se parece á los de la torre de Babel, en un país cuya efigie heterogénea se revela en las costumbres, en la sociabilidad y en las artes. (Sicardi, 1899: 25).

\section{Conservación y desplazamientos}

En esta compleja situación de contactos lingüísticos, el horizonte identitario de los sujetos y de las comunidades se construye en una frontera múltiple y heterogénea que permite la reelaboración y la redefinición de pertenencias y alteridades. En ese espacio, caracterizado por la continua búsqueda de equilibrio, la fragmentación, los desplazamientos, las cristalizaciones, las sustituciones, las reactualizaciones tratan de resolver los conflictos de extranjeridad, de lejanía y de contrastes. En algunos textos las representaciones de los contactos lingüísticos relatan la conservación de una identidad de origen, incluso por mandato familiar. Así, Ferdinando Resasco en Alle rive del Plata. Ricordi di viaggio (1890) narra que, al llegar a Buenos Aires, reconoce el dialecto genovés, que él también habla en cuanto originario de esa ciudad, en la forma de hablar del facchino y, creyéndolo un emigrante, le pregunta si es de Génova. La sorpresa para Resasco se produjo al saber que el portero había nacido en América, también como su padre, por lo que el dialecto se conservaba sin alteraciones en la tercera generación de emigrantes. Esta anécdota (Resasco, 1890: 131) muestra, entonces, la conservación y transmisión intergeneracional del dialecto y de las formas culturales y simbólicas de pertenencia, así como la realización de la ley de Retorno de la Tercera Generación de Marcus Lee Hansen (1938). Edmondo De Amicis en "I nostri contadini», incluido en In America (1897) resalta, en la descripción de las colonias, el uso de piamontés y del lombardo como lenguas de comunicación (De Amicis, 1897: 61) y la comprensión de las colonias, para los emigrantes, como una extensión extraterritorial de su Patria. La lengua franca en las colonias no resultaba, según el relato de De Amicis, el español ni el italiano sino el piamontés, pues 
era la lengua utilizada en los negocios incluso por miembros de otras comunidades. De esta forma el encuentro de De Amicis con los colonos representó una continuidad con la comunidad regional, sin que se plantearan fragmentaciones o tensiones:

Ora non è più in America; è nel suo paese, in Piemonte; anzi, in familia. - Vedrà - dicevano - la colonia di San Carlos. Là siamo tutti patiotti, migliaia di piemontesi, la più bella colonia di Santa Fe. Lo condurremo domani all'uscita della messa grande. - Migliaia di piemontesi, in fatti; nel consiglio comunale si parla piemontese; i tedeschi, gli inglesi, i francesi che hanno affari con la colonia, bisogna che imparino il dialetto, e lo imparano. (De Amicis, 1897: 61-62)

En Emigrati. Studio e racconto (1880-1881) de Antonio Marazzi, las representaciones del uso y de la conservación de los dialectos funciona no solamente como localización de los emigrantes, sino también como índice de una continuidad identitaria que produce una ruptura de las expectativas de (re)conocer la alteridad. Así, en vez de lo extraño se presentaba, en el nuevo espacio americano, lo conocido que, descontextualizado y en contraste con las expectativas frente al nuevo mundo, se conformaba como un próximo lejano. Dos anécdotas, en el primer volumen de Emigrati. Studio e racconto, relatan el encuentro de los protagonistas con otros italianos, delineando el extrańamiento y el asombro al reconocer la presencia italiana en América. El «encuentro» con un genovés, camino a la colonia, que los echa de su rancho cuando solicitan alojamiento (Marazzi, 1880: 302) y con los marineros marcan la ruptura de las expectativas de extrañamiento paradójicamente a través del reconocimiento lingüístico:

—Oh beate anime dei miei poveri morti! Sono dei nostri!... sclamò esterefatto il Codazzi, che s'aspettava di aver a che fare con gente di razza sconosciuta, parlante una lingua per lui affatto nuova. Erano infatti alcuni marinai appartenenti a diverse provincie italiane, che trovando pel momento minor convenienza a navigare l'Oceano che a fare il carbonajo, s'erano dati a quest'ultimo mestiere. (Marazzi, 1880: 275)

La conservación (o no) de la lengua materna, sea el dialecto o el italiano, es una constante en las representaciones y discursos sobre la emigración italiana en Argentina. Su desplazamiento o conservación dependía, entre otros factores, de la presencia femenina en el núcleo familiar, como señaló Giovanni Graziani:

Il marito, italiano, costretto da mille necessità a vivere in mezzo all'ambiente nuovo, ne assorbe a poco a poco tutte le caratteristiche ed è costretto a parlare sempre la lingua nazionale, ch'è la spagnuola, anche in casa, dove la moglie, creola, dal canto suo, nella consuetudine coniugale, cancellerà a poco a poco anche quelle ultime traccie d'italianità rimaste nel marito.

Se invece la donna di casa è una italiana, essa, che lontana dalle brighe dei pubblici negozi, vive delle gioie della familia e dei sacri ricordi della giovinezza, saprà invece infondere fin dai primi anni nei figlioletti l'amore alla patria lontana, parlerà loro delle sue bellezze e delle sue glorie (...) (Graziani, 1905: 82)

Por su parte, Aníbal Latino (José Ceppi) en Lejos del terruño (1905) describe la formación individual de interlenguas, a partir de los residuos deformados y cristalizados del dialecto, del italiano, adaptados al español. Se trata de una fragmentación de los códigos lingüísticos, por oscilaciones, debido a los contactos y a la voluntad de comu- 
nicación, amplificados por la debilidad gramatical y la oralidad. La lengua con más prestigio desplazaba las otras, organizándose en estratificaciones y superposiciones:

Si no fuese cosa impalpable, si fuese cosa visible como lo es perceptible para el oído el lenguaje que al cabo de algunos ańos de residencia en América, hablan los italianos del vulgo, que ya cuando vienen no saben otra cosa que su dialecto, si pudiese recogerse como los fósiles, los animales y otras cosas de la naturaleza, no habría, sin duda, en los museos nada más extraño ni más digno de verse. Ya no es la lengua ni el dialecto lo que hablan, sino una mezcla bizarra y original de lenguas y dialectos, un lenguaje lleno de palabras contrahechas, cuyo origen y filiación se adivinan, pero que no pertenecen propiamente á país alguno. Sobre el fondo primitivo de su dialecto, que más ó menos siempre predomina, cada italiano sin instrucción agrega palabras españolas, criollas y de los demás dialectos de Italia, corrompiéndolas ó amoldándolas á su pronunciación peculiar. Por eso en la clase baja no se hallarán dos italianos que hablen igual en criollo, cuando pretenden hablarlo, como sucede generalmente. (Latino, 1905: 113)

Francesco Scardin, en cambio, en su Vita italiana nell'Argentina. Impressioni e note (1899) resalta el trabajo de las escuelas italianas en Argentina para la conservación y difusión del italiano valorizando el haber «introdotto nelle scuole della Repubblica Argentina l'insegnamento della lingua italiana, come si è fatto di altri idioma stranieri, meno vitali ed importante, per l'Argentina, dell'idioma nostro" (Scardin, 1899: 171). En relación con estas escuelas, explicó que muchos italianos enviaban a sus hijos a fin de evitar las pérdidas, las interlenguas y los desplazamientos lingüísticos, en resumen, como una forma de mantener un vínculo con la tierra y la cultura de origen.

\section{Mínimas conclusiones}

Este recorrido por algunas representaciones significativas de los contactos lingüísticos permite reconocer algunos genotextos y problemas claves vinculados con los desplazamientos, los conflictos, los reacomodamientos identitarios. La visibilización y abordaje en los textos indica su centralidad en las reflexiones sobre la e(in)migración italiana en Argentina, declinados desde diferentes perspectivas ideológicas, no necesariamente coincidentes. Por otra parte, en cuanto son discursos culturales que pueden leerse desde su literaturidad o no, el corpus asume una valencia documental que permite reconstruir un estado de consideraciones y posiciones frente a esas realidades, y también delinear un panorama de los discursos y de los mecanismos socio-culturales. Las representaciones y estilizaciones resultan, entonces, análisis de las re/de-construcciones identitarias del e(in)migrante que, como señala Sonia Floriani,

(...) consapevole della sua condizione esistenziale deprivata del senso di appartenenza e di quello di consistenza e di continuità, organizza strategicamente le sue risorse esperienziali al fine di contrastare il senso di estraneità o quello di frantumazione biográfica, e di ridefinire le appartenenze o di ricomporre i frammenti del suo vissuto. (Floriani, 2004: 92) 


\section{Notas}

${ }^{1}$ Este trabajo recoge algunos resultados del Proyecto de Investigación «Incidencia de la cultura italiana en la configuración del imaginario argentino» del Consejo Nacional de Investigaciones Científicas y Técnicas (CONICETArgentina) con sede en el Instituto de Literatura Argentina «Ricardo Rojas» de la Facultad de Filosofía y Letras de la Universidad de Buenos Aires. Otros resultados relativos a las representaciones de los conflictos lingüísticos en vinculación con la e(in)migración italiana en la Argentina ya han sido presentados en trabajos anteriores (Bravo Herrera, 2014, 2015, 2017a, 2017b). ${ }^{2}$ Son numerosos los estudios que abordan las representaciones lingüísticas y dialectales en la escritura de Edmondo De Amicis, sea en su producción narrativa como en la periodística. Entre los ensayos que pueden mencionarse, entre otros, los estudios de Franco Pierno (2012), Lorenzo Tomasin (2012) y Matteo Grassano (2018).

${ }^{3}$ Se incluye la cita de Sull'Oceano, aunque extensa, en cuanto la enumeración resulta oportuna para reconstruir un panorama del componente demográfico de la emigración italiana hacia América del Sur en la mitad del siglo XIX: «La maggior parte degli emigranti, come sempre, provenivano dall'Italia alta, e otto su dieci dalla campagna. Molti Valsusini, Friulani, agricoltori della bassa Lombardia e dell'alta Valtellina: dei contadini d'Alba e d'Alessandria che andavano all'Argentina non per altro che per la mietitura, ossia per metter da parte trecento lire in tre mesi, navigando quaranta giorni. Molti della Val di Sesia, molti pure di que' bei paesi che fanno corona ai nostri laghi, così belli che pare non possa venir in mente a nessuno d'abbandonarli: tessitori di Como, famigli d'Intra, segantini del Veronese. Della Liguria il contingente solito, dato in massima parte dai circondari d'Albenga, di Savona e di Chiavari, diviso in brigatelle, spesate del viaggio da un agente che le accompagna, al quale si obbligano di pagare una certa somma in America, entro un tempo convenuto. Fra questi c'erano parecchie di quelle nerborute portatrici d'ardesie di Cogorno, che possono giocar di forza coi maschi più vigorosi. Di Toscani un piccolo numero: qualche lavoratore d'alabastro di Volterra, fabbricatori di figurine di Lucca, agricoltori dei dintorni di Firenzuola, qualcuno dei quali, come accade spesso, avrebbe forse un giorno smesso la zappa per fare il suonatore ambulante. C'erano dei suonatori d'arpa e di violino della Basilicata e dell'Abruzzo, e di quei famosi calderai, che vanno a far suonare la loro incudine in tutte le parti del mondo. Delle province meridionali i più erano pecorari e caprari del litorale dell'Adriatico, particolarmente della terra di Barletta, e molti cafoni di quel di Catanzaro e di Cosenza. Poi dei merciaiuoli girovaghi napoletani; degli speculatori che, per cansare il dazio d'importazione, portavano in America della paglia greggia, che avrebbero lavorata là; calzolai e sarti della Garfagnana, sterratori del Biellese, campagnuoli dell'isola d'Ustica. In somma, fame e coraggio di tutte le province e di tutte le professioni, ed anche molti affamati senza professione, di quelli aspiranti ad impieghi indeterminati, che vanno alla caccia della fortuna con gli occhi bendati e con le mani ciondoloni, e son la parte più malsana e men fortunata dell'emigrazione». (De Amicis, 1996: 20-21) 


\section{Referencias bibliográficas}

Barzini, L. (1902). L'Argentina vista come è. Milano: Corriere della Sera. Blomberg, H.P. (1920). Las puertas de Babel. Buenos Aires: Cooperativa Editorial Limitada - Agencia general de Librería y Publicaciones. Bravo Herrera, F.E. (2014). «E(in)migración italiana en la Argentina y conflictos lingüísticos. Representaciones literarias y variaciones en las dos orillas». Revista del Instituto de Investigaciones Lingüisticas y Literarias Hispanoamericana (RILL), 19 (1), 60-85. San Miguel de Tucumán: Insil, Departamento de Publicaciones, Facultad de Filosofía y Letras, Universidad Nacional de Tucumán.

(2017a). «Espacios, fronteras y conflictos lingüístico-culturales. Representaciones de la e(in)migración italiana en la Argentina». Oltreoceano. Rivista sulle migrazioni (13), 173-183. Udine: Forum Oltreoceano, Centro Internazionale Letterature Migranti, Università degli Studi di Udine.

— (2017b). "Humor y representaciones de la e(in)migración italiana en la Argentina» en Cusato, D.A. y Galzio, C. (Coord.), Ricordando Antonio Melis. Omaggio degli amici di Testo, Metodo, Elaborazione elettronica. Salem - Lima - New York: Axiara Editions, pp. 41-52.

(2015). Huellas y recorridos de una utopía. La emigración italiana en Argentina. Buenos Aires: Teseo.

Bufalino, G. (2006). Opere/I (1981-1988). Milano: Bompiani.

Ceppr, J. (Aníbal Latino) (1888). Cuadros sud-americanos. Buenos Aires: Librería Universal de Alejandro Miroli, Librero Editor.

De Amicis, E. (1996). Sull'Oceano. Milano: Garzanti.

- (1905). L'idioma gentile. Milano: Fratelli Treves editori. (1897). In America. Roma: Enrico Voghera.

Floriani, S. (2004). Identità di frontera. Migrazione, biografie, vita quotidiana. Soveria Mannelli: Rubbettino.

Gálvez, M. (1920). «Prólogo» en Blomberg, H.P., Las puertas de Babel. Buenos Aires: Cooperativa Editorial Limitada - Agencia general de Librería y Publicaciones, pp. 7-12.

Gramsci, A. (1977). Quaderni dal carcere. Torino: Einaudi.

Grassano, M. (2018). La prosa parlata. Percorsi linguistici nell'opera di Edmondo De Amicis. Milano: FrancoAngeli.

Graziani G. (1905). La emigrazione italiana nella Repubblica Argentina. Opera corredata da recentissimi dati statistici seguita da numerosi allegati e da ricca notizia bibliografica. Torino-Roma-Milano-Firenze-Napoli: Ditta G.B. Paravia e Comp.

Hansen M. L. (1938). The Problem of the Third Generation Immigrant. Rock Island: Augustuna Historical Society.

Latino, A. (1905). Lejos del terruño. Barcelona: Casa Editorial Maucci / Buenos Aires: Maucci Hermanos.

(1914). El concepto de la nacionalidad y de la patria. Valencia: Editorial Prometeo.

Marazzi, A. (1880). Emigrati. Studio e racconto. Vol. I Dall'Europa in America. Milano: Fratelli Dumolard. 
Lenguas en desplazamiento · F. Bravo Herrera

Pierno, F. (2012). “Ah, povra Italia!”. Appunti su dialetto e rappresentazioni linguistiche en Sull'Oceano» en Polimeni, G. (Coord.) (2012), L'Idioma gentile. Lingua e società nel giornalismo e nella narrativa di Edmondo De Amicis. Pavia: Edizioni Santa Caterina, pp. 105-148.

Poletti, S. (1972). Gente conmigo. Buenos Aires: Losada.

Resasco, F. (1890). Alle rive del Plata. Ricordi di viaggio. Milano: Fratelli Treves Editori.

SANFilippo, M. (2005). Problemi di storiografia dell'emigrazione italiana. Viterbo: Sette Città.

SCARDIn, F. (1899). Vita Italiana nell'Argentina. Impressioni e note. Buenos Aires: Compañía Sud-Americana de Billetes de Banco.

Tomasin, L. (2012). «De Amicis tra riflessione e prassi linguistica» en Lingua nostra (vol. LXXIII), 92-101.

\section{Bravo Herrera, Fernanda}

«Lenguas en desplazamiento. Representaciones literarias de problemáticas lingüísticas en la e(in)migración italiana en Argentina». El hilo de la fábula. Revista anual del Centro de Estudios Comparados (19), 153-166. 